\title{
Designing an Adaptive 3D Body Model Suitable for People with Limited Body Abilities
}

\author{
Kozar T*, Rudolf A, Cupar A, Jevšnik S and Stjepanović Z
}

Faculty of Mechanical Engineering Department of Textile materials and Design, University of Maribor, Slovenija

\begin{abstract}
The purpose of this research was to develop a generalized adaptive 3D body model the posture of which could be adapted to different positions in order to perform a virtual prototyping of garments for people with limited body abilities. The digital data of a tested person's body was acquired using the GOM Atos scanning system. Careful surface reconstruction was performed in order to provide an adequate mesh for further posture adaptation. The experimental part of this article presents the usages of a variety of graphic programs in order to provide an adaptive 3D body model through kinematic skeleton construction. The mesh-deformation during posture adaptation was improved using advanced tools of applied software packages. The usefulness of the gained 3D body model was determined by calculating the differences between the real and digital 3D body model measurements.
\end{abstract}

Keywords: Scanning technology; 3D body model; 3D body model posture adaptation; People with limited body abilities

\section{Introduction}

The utilities of three-dimensional body models, also called avatars or humanoids, are unlimited just as human activities are unlimited. The book "Virtual Humans" clearly demonstrates the scope of their applicability from virtual presenters for Television (TV) and World Wide Web (WEB), to virtual assistants for training in the case of emergency, virtual workers in industrial applications, virtual actors in computer-generated movies to virtual characters for the garment industry [1]. The complexity of today's garment industry has led to unit advanced computer-aided (CAD) technologies and 3D graphic software for assisting designers' creativities, reducing garments' manufacturing costs and more importantly to serve customers' needs and increase their satisfaction percentage [2]. Recently, an obvious trend in garment manufacturing processes has become epitomized by individualized garment production supported by CAD systems for garment pattern construction [3-6] and their appearances on parametric body models in standing positions, the body dimensions of which could be adjusted to specific customers' body measurements. It refers to garment virtual prototyping based on a made-to-measure concept for ensuring garments' pattern adaptation by taking individual requirements for a garment's style and functionality into consideration [7]. It is wellknown that the use of 3D CAD systems for garment pattern design has decreased the production of real garments' prototypes by $20 \%$ and the amount of manufacturing waste [8]. A major issue regarding garment virtual prototyping is when evaluating a garment's fit appearance and the fabric's draping behavior on virtual body models are limited to a standing posture with standard body shape characteristics. These body shapes may be adaptable for healthy individual body measurements but useless if there is a need to construct individualized garments for people with physical disabilities.

Based on the United Nation's definition, persons with physical disabilities include those who have long-term physical, mental, intellectual, or sensory impairments, which during interactions with various barriers may hinder their full and effective participation in society on an equal basis with others [9]. In our presented contribution we focused on people with limited body abilities, caused by several forms of injuries, diseases, and amputations. This group of people within the world's population have different kinds of limitations and health conditions and therefore these people with limited body abilities are sensitive about their garments and general appearances. Therefore, greater attention should be focused on constructing special needs garments, a category of functional garments for persons with physical limitations, whose body shapes, sizes, mobility or dexterity are significantly different from people with average body shapes [10]. While the application field of $3 \mathrm{D}$ body models is unlimited and improvements and progressions are focused on imitating real humans' behavior and appearance within virtual environments, there is need to observe an adaptive $3 \mathrm{D}$ body model and thereby adaptive garment prototyping for improving the qualities of life of this special group of people.

To date several techniques for human body modeling have been devoted to automatic modifications resulting in models of different shapes with the help of example models [11,12]. One researcher developed a method for fitting high-resolution template meshes to detailed human body range scans from the CEASAR dataset. In order to find a consistent shape representation of non-structured data, this researcher observed two surface-matching algorithms for creating a shape model that could generate different kinds of body shapes in different poses but with limitations to the skinning framework, showing a non-linear relation to the proportion of the body at the joint angles [13]. The parameterization approach synthesizes sample models to a new body model according to the input sizing dimensions. Along with synthesizing the system is characterized by feature wireframes of scanned human bodies for enabling garment design [14]. In addition, there are several methods for fitting deformable body models to body shapes, as parameters to ensure a variety of body shapes $[15,16]$. Other methods have used data-driven models where the variation of human body shapes was based on a training set of 3D body shapes

*Corresponding author: Kozar Tatjana, Faculty of Mechanical Engineering Department of Textile materials and Design, University of Maribor, Slovenia, Tel: +386 30367 762; E-mail: tatjanka.kozar@gmail.com

Received July 11, 2014; Accepted August 11, 2014; Published August 25, 2014

Citation: Kozar T, Rudolf A, Cupar A, Jevšnik S, Stjepanović Z (2014) Designing an Adaptive 3D Body Model Suitable for People with Limited Body Abilities. J Textile Sci Eng 4: 165. doi:10.4172/2165-8064.1000165

Copyright: ( $) 2014$ Kozar T, et al. This is an open-access article distributed under the terms of the Creative Commons Attribution License, which permits unrestricted use, distribution, and reproduction in any medium, provided the original author and source are credited. 


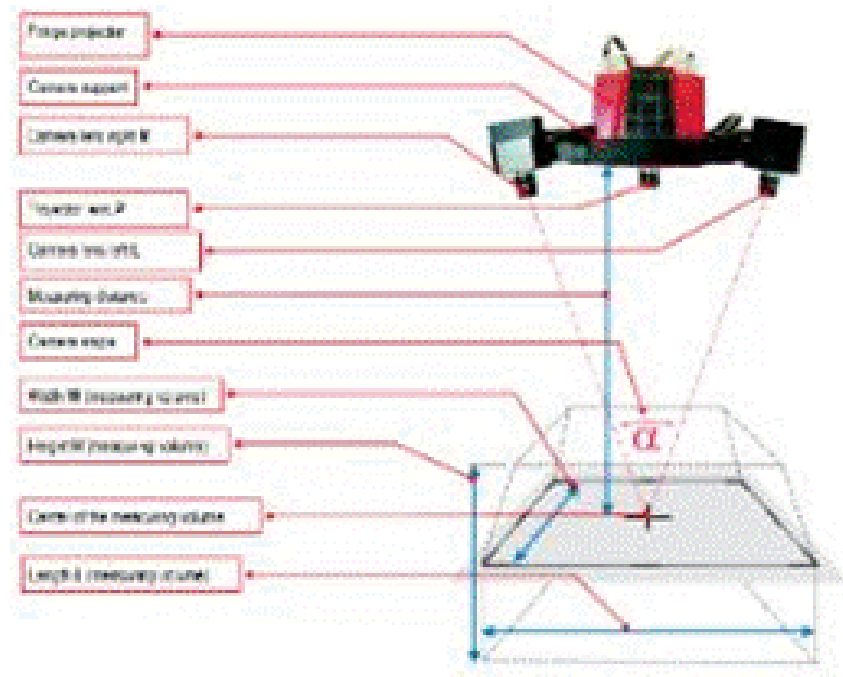

Figure 1: GOM Atos II optical scanner schematics.

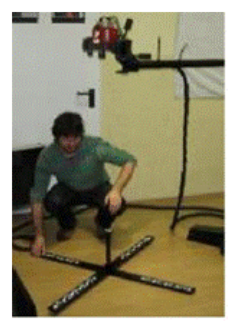

(a)

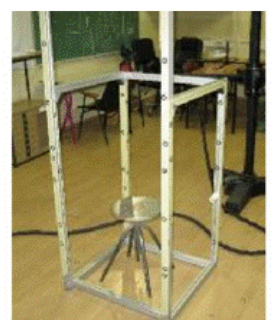

(b)

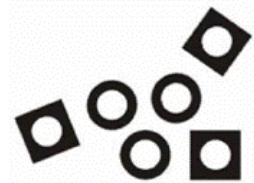

(c)
Figure 2: Calibration of optical scanning system GOM Atos II 400

[17]. Obviously, the mentioned human body modelling techniques are focused on a standard standing posture, involving healthy individuals and exclude persons with physical limitations.

Over recent years, certain research has been devoted to acquiring an accurate 3D body model in a sitting posture for garments' virtual prototyping. Two types of scanning technologies have been applied for obtaining a suitable three-dimensional image of a human body in a sitting posture [18,19]. Firstly, the Vitus Smart scanner was introduced for acquiring a polygonal meshed surface of a test person in a sitting posture in order to achieve a garment's virtual prototyping and its fit appearance. Its technical features, operation principle and meshprocessing are clearly described in [18]. Next, scanning of a human body in a sitting position by using a GOM ATOS scanner was introduced and performed with the help of a specially developed frame with hand rails [19]. Finally, by analyzing a 3D body model's measurements in both standing and sitting positions, the researcher assessed the fit of the garments intended for paraplegics and took terms of wearing comfort on specific body parts into consideration [20]. The GOM Atos scanning system's primary use refers to the exploration of an object's digital data within the field of mechanical and civil engineering. Its accuracy was analyzed in a study [21], where five digitization techniques like laser scanners, one fringe projection system and one $\mathrm{x}$-ray system on calibrated objects, with dimensions of less than $300 \times 500 \mathrm{~mm}$ were compared. Another research was oriented towards finding a suitable 3D scanner for the digitization of human body parts within the field of prosthetics and orthotics. Due to higher resolution features and higher requirements for preparing the scanning process, the scanning systems ATOS I and ATOS Triple Scan predominate among other tested scanners when scanning a plaster cast among other tested scanners, while the tested hand scanner Artec $\mathrm{MH}$ showed a better alternative for a direct scanning of a patient's body parts [22].

In order to increase the interest of people with limited body abilities within the garment manufacturing process, the aim of this study was to develop a generalized adaptive 3D body model within the following activities:

- Scanning the surface of a test person's body in standing and sitting positions.

- Reconstruction of observed polygonal meshed surfaces.

- Kinematic skeleton construction inside a watertight polygonal mesh in a standing position.

- Adjusting the $3 \mathrm{D}$ body model from a standing to a sitting position.

- Comparing the real body measures with a 3D body model's measures in standing-, sitting- and adjusted sitting positions.

\section{Experimental}

\section{Scanning of a human body with a GOM Atos II 400 3D optical scanner}

In the field of computer graphics for human body modeling, scanning technology represents an effective technique for obtaining the polygonal meshed surface of a human body in order to avoid manual sculpting techniques of meshed surfaces. In this research, the surface of a human body in both standing and sitting positions was obtained by using a three-dimensional laser scanner.

The GOM AtosTM II 400 [23], is installed at the Faculty of Mechanical Engineering in Maribor and used for different research purposes. Figure 1 shows a schematic representation of a GOM Atos scanning system, which operates using a triangulation principle $[20,21]$, for converting the obtained point clouds to three- dimensional surfaces. Its configuration includes the following technical features: measuring volume of $1200 \times 960 \times 960 \mathrm{~mm}^{3}(\mathrm{~L} \times \mathrm{W} \times \mathrm{H})$, measuring point distance of $0.94 \mathrm{~mm}, 6 \mathrm{~mm}$ projector lens and $8 \mathrm{~mm}$ camera lens.

The GOM Atos scanning system uses white light for obtaining polygonal meshed surfaces, when different fringe patterns, recorded by two cameras, are projected onto the scanned object - in our case on a human body through the sensor units. Garment pattern construction of a tight suit was established for observing accurate shape information from a tested person's body. Generally, such a scanning method is an accepted procedure for scanning human bodies which does not particularly differ from scanning human bodies wearing underwear. The digital body information of a scanned human body was obtained in three steps. First, the measuring system was calibrated with a calibration cross, (Figure 2a) to ensure the system's dimensional consistency. Next, the test person was scanned in both standing and sitting postures from different heights and angles, while each measurement generated up to 4 million data points. In order to avoid incorrect alignment of individual scans in a sitting posture, a frame with hand rails was used to improve the static position of a human body. The frame was marked with reference points or circular markers in order to improve the process of alignment (Figure $2 \mathrm{~b}$ ). Self-adhesive markers had a defined geometry (white Circle on a black background) (Figure 2c), and served 


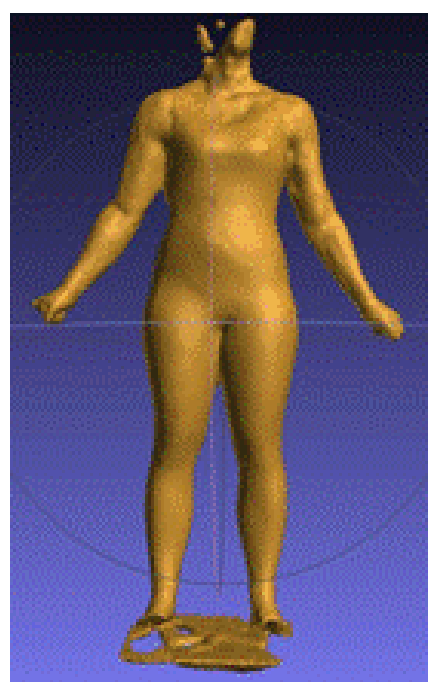

Figure 3: Aligned individual scans in a global coordinate system using Atos V6.0.2 software.

as connection points for the individual measurements to provide their transformation into a common coordinate system. Therefore, we finally used the Atos V6.0.2-6 software, where each scan was polygonized into one independent mesh in order to perform further fit registration (Figure 3).

\section{Scanned surface processing}

The polygonal meshed surfaces, obtained by general 3D laser scanners, represent in many cases starting points for further computer modelling and visualization of physical objects. In order to ensure the body model's appropriate fitting by taking into account the real subjects, we obtained an optimal surface description through preprocessing and post-processing procedures, which undoubtedly stood out as the more significant and challenging tasks in obtaining the digital model from the physical object [24]. Table 1 clearly demonstrates a short description of applied programs and indicates the applied tools for performing surface-processing procedures.

The pre-processing procedure was carried out by using the GOM Inspect program. GOM Inspect is a $3 \mathrm{D}$ inspection and mesh processing software for three dimensional analysis of 3D point clouds, obtained from white light scanners, laser scanners, CT's and other sources. The program is independently tested and certified by national measurement laboratories, such as German PTB (Physikalisch-Technische Bundesanstalt) and American NIST (National Institute of Standards and Technology) [25]. We performed the noise reduction through cleansing and removing those points which did not correspond to the body surface, like the digital data of a chair from the point data set shown in Figure 4a. The resulting holes are shown in Figure 4b, which were repaired manually through modeling and adding new surfaces, Figure 4 . The obstructed areas were repaired as also were the scanner had failed to capture any data, especially at the thigh- and calf areas. Consequently, the tool "Mesh Bridge" was used to reduce mesh errors.

In addition, pre-processed polygonal meshes usually need higher refinements and the surface was reconstructed through a postprocessing procedure which was also done manually. In addition, graphic programs like MeshLab and Blender were used to correct imperfections and errors on the surface. Meshlab is an open source, portable and extensible system for the processing and editing of unstructured 3D triangular meshes, providing a set of tools for editing, cleaning, healing, inspecting, rendering and converting of this kind of meshes. The system is based on the VCG Library (The Visualization and Computer Graphics Library) developed at the Visual Computing Lab of ISTI-CR (Instituto di Scienza e Technologie dell' Informazione) in Italy [26]. During the first step we created a new average mesh over the existing scan by using the tool "Select All-Filter Selection" from the program MeshLab. Afterwards, the surface was reconstructed using the tool "Poisson", Figure 6.

Partially reconstructed polygonal meshes were imported in the form of .stl files into the Graphic Program Blender for smoothing the remaining irregularities on the meshed surface. The tool "Sculpt ModeSmooth" was used to provide an entirely watertight mesh (Figure 7).

Although it was necessary to reconstruct the mesh at some areas, especially at the region after removing the scanned data of a chair, the corrections do not affect the actual shape of the body. It is evident that the body shape of the reconstructed 3D body model in

\begin{tabular}{|c|c|c|}
\hline Applied program & Features Description & Applied Tools \\
\hline GOM Inspect & $\begin{array}{l}\text { 3D inspection and mesh processing } \\
\text { software for editing } 3 D \text { point clouds, } \\
\text { containing evaluation and editing tools } \\
\text { for an extensive analysis of parts and } \\
\text { components. }\end{array}$ & $\begin{array}{l}\text { "Delete } \\
\text { Neighborhood", } \\
\text { "Mesh Bridge" }\end{array}$ \\
\hline MeshLab & $\begin{array}{l}\text { 3D mesh processing software for editing } \\
\text { unstructured 3D triangular meshes, } \\
\text { containing editing filters for cleaning } \\
\text { meshes, remeshing and mesh inspections. }\end{array}$ & $\begin{array}{l}\text { "Select All-Filter } \\
\text { Selection". } \\
\text { "Poisson" }\end{array}$ \\
\hline Blender 2.71 & $\begin{array}{c}\text { Open source 3D graphics and animation } \\
\text { software, enabling 3D modeling, rigging, } \\
\text { sculpting, rendering and interactive creation } \\
\text { of 3D models. }\end{array}$ & $\begin{array}{l}\text { "Sculpt Mode- } \\
\text { Smooth" }\end{array}$ \\
\hline
\end{tabular}

Table 1: Applied programs and tools for surface reconstruction.

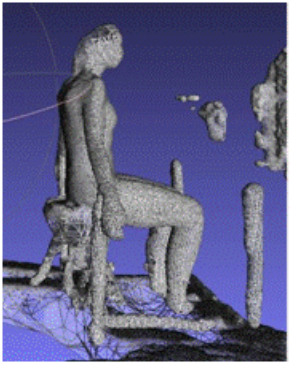

(a)

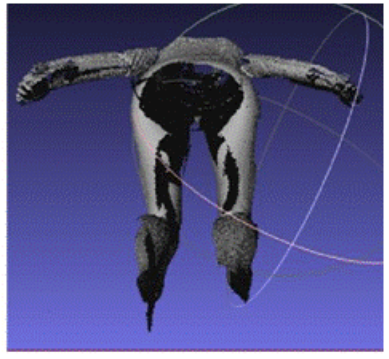

Figure 4: Mesh errors after removing the digital data of a chair by using the tool "Delete Neighborhood".

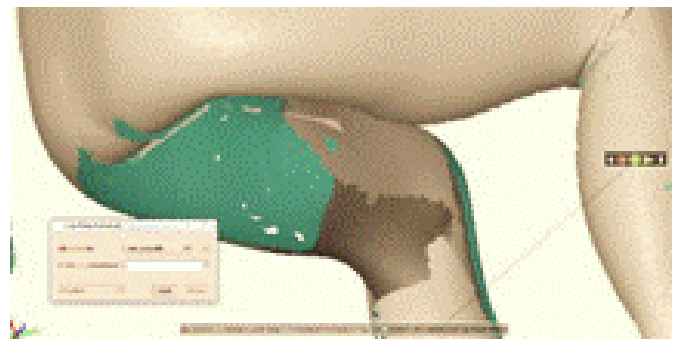

Figure 5: Applying the "Mesh Bridge" tool for surface modeling. 


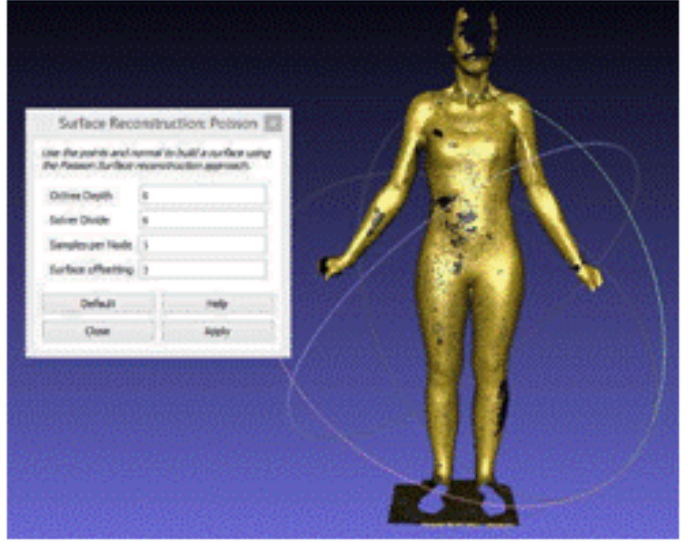

Figure 6: Removing the remaining holes in Meshlab by using the tool "Poisson".

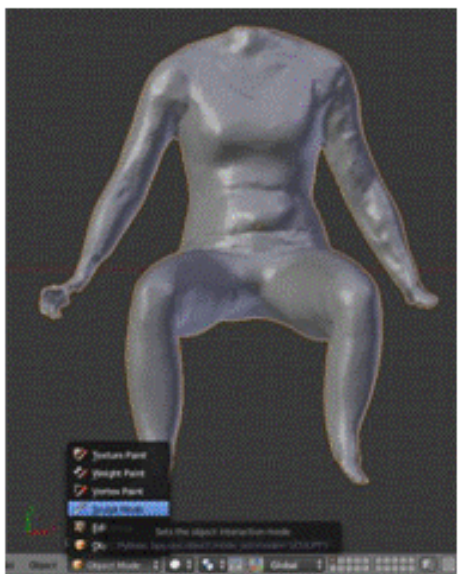

Figure 7: Program Blender for the smoothing of noise and irregular polygons on a polygonal mesh.
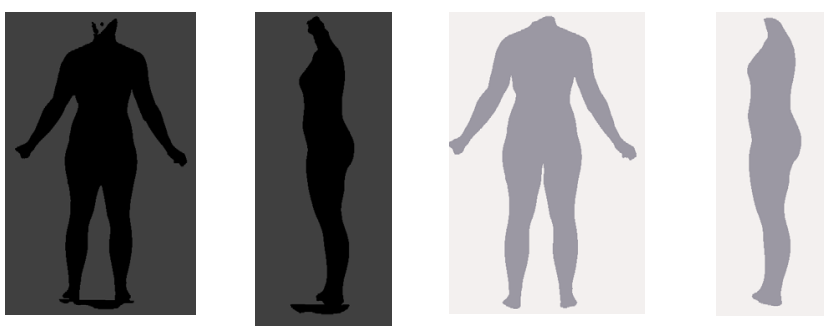

Figure 8: Extracted body silhouettes of observed body model after scanning (a) and extracted body silhouettes of a reconstructed 3D body model (b) in a standing position, from a front and side view.

a standing position; (Figure 8a) does not significantly differ from the observed body model after scanning procedure (Figure $8 \mathrm{~b}$ ). The same applies to the visual assessment of differences between body shapes of the reconstructed 3D body model and the observed body model after scanning procedure (Figures 9a and Figure 9b).

\section{Kinematic skeleton construction}

A related study discussed 3D body models' posture adaptations through the kinematic skeleton construction of 19 bones and 15 joints [27]. Pants- and T- shirt patterns construction was carried out in order to simulate a garment's behavior and virtual fitting using the 3D body model in a sitting position. Its accuracy was tested by measuring the virtual body measurements and comparing them with a real person's body measurements in a sitting position. An additional indicator when calculating surface areas in square centimeters was used after adapting a 3D body model to images taken from two different angles. Differences between the body measurements were encountered at the hips, and under the knee and calf circumferences. Even then there were no larger differences between the silhouettes extracted from the images, the visual assessment displayed clear differences between the extracted silhouettes. In order to provide a generalized adaptive 3D body model, which should imitate the real human's behavior and ensure accurate anthropometric measurement of those three-dimensional body measurements in a sitting position, the kinematic skeleton construction inside the observed polygonal mesh was improved by taking specific anatomical landmarks into consideration. Anthropometry, as one of the methods within anthropology, studies human body measurements and the proportions between different parts of the body [28] beginning with accurate placements of anatomical landmarks or feature point on the human body's surface that indicate the locations of body organs or components [1]. In order to ensure accurate anthropometric measurements of the three-dimensional body measurements, we learned from the H- Anim standard [29] and used the H-Anim feature point's definition as a basic guide line for constructing the kinematic skeleton. In regard to the skeleton construction inside the watertight mesh of a scanned body in a standing position, we used the program Blender 2.68. Blender 2.68 is open-source 3D graphic software, as maintained by the Blender foundation [30] which assists designers with similar functions and animation tools as commercial software. Its suitability for creating virtual humans with realistic human animations was proved during the study [31] where the researcher extended the Blender's functionality for computer vision applications. In our contribution, we built an articulated 3D body model consisting of a polygonal mesh and a kinematic skeleton. The observed polygonal mesh of vertices, edges, and faces or polygons, describes the highly complex geometric structure of a scanned human. Each polygon is defined by 3 or more vertices with $\mathrm{x}, \mathrm{y}$, and $\mathrm{z}$ coordinates within a 3D space. For the skeleton construction we imported the 3D body model in a standing posture in the form of a .stl file into the program Blender. The "Armature Modifier" was used to construct a hierarchical skeleton of 20 bones and 15 joints (Figure 10).

In Blender the term "Armature" refers to an object that deforms a mesh model and it borrows many ideas from real life skeletons. In
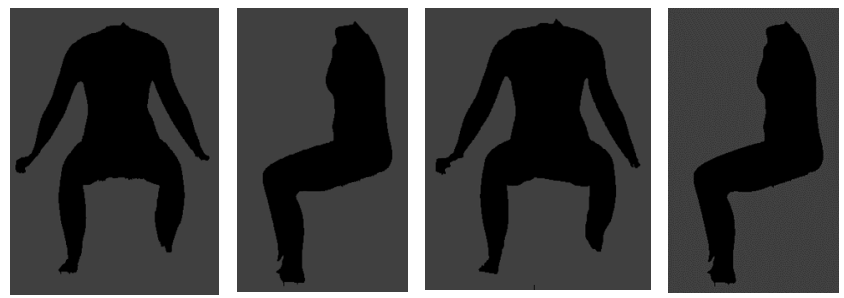

Figure 9: Extracted body silhouettes of observed body model after scanning (a) and extracted body silhouettes of a reconstructed 3D body model (b) in a sitting position, from a front and side view. 


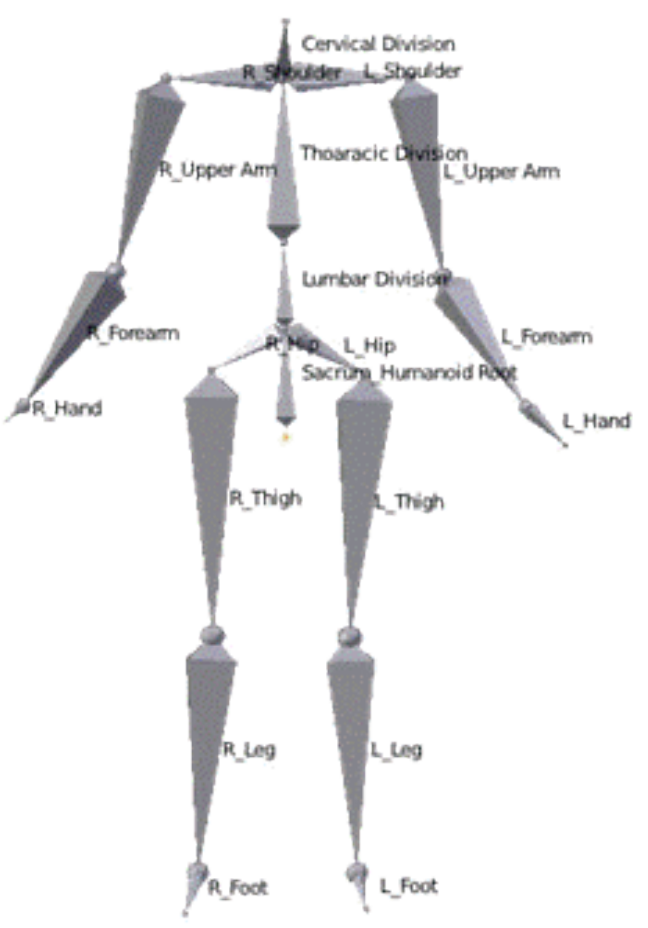

Figure 10: Rendering the skeleton construction in the Blender's "Top View".

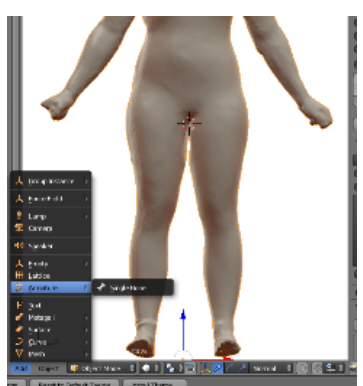

(a)

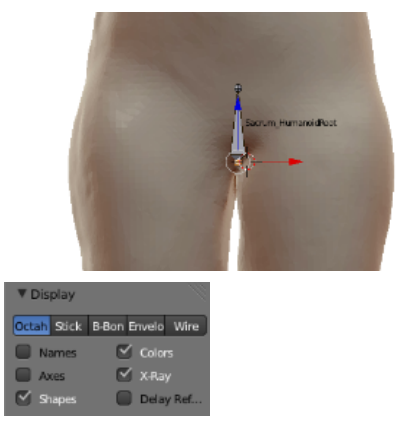

(b)
Figure 11: Blender's »Armature modifier« for skeletal construction.

"object mode" with a selected mesh of a 3D body model the 3D Cursor was placed at the crotch point. By adding the "Armature Modifier", the skeleton construction started up with a single bone, called the "Sacrum_ Humanoid Root" or the base- bone of a skeleton's hierarchical structure (Figure 11). In the "Properties Window" an "Object button" was used which makes the bone visible using the option X-Ray (Figure 11b).

Firstly, in "Edit Mode" the spinal cord construction was carried out by selecting the tail of each particular constructed bone through extruding it to a new bone (Figure 12a). The skeleton construction continued for the shoulder and upper limbs at the left and right sides shown in Figure 12b, and completed at the lower limb for the Left and Right sides in Figure 12c.

In order to provide effective posture adaptation, it was necessary to establish a "Parent-Child" relationship between the constructed bones. For example, in "Edit Mode" with the bone head and the bone

tail selected, the "Lumbar Division" bone was added by extruding its "Parent bone", which parents it to the constructed bones automatically, (Figure 13).

For example, all the bones of the spinal cord, from the "Lumbar Division" bone to the "Cervical Division" bone, have their rotational centers at their parent bones. This means that the "Cervical Division" bone is the child of the lower bone, called the "Thoracic Division" bone, which is further the child of the "Lumbar Division" bone, which is further the child of the "Sacrum Humanoid Root" base-bone. This means if we rotate or move the lower bone, its child will also be affected. However, if we rotate or move a child bone, its parent bone will not be affected.

Further, the head or the tail of each particular bone represents the location of an anatomical feature, the position of which has to be located by visual assessment of extreme feature points from the side view of the body and by taking specific anthropometric rules into consideration. The anatomical feature points are summarized and defined in Table 2.

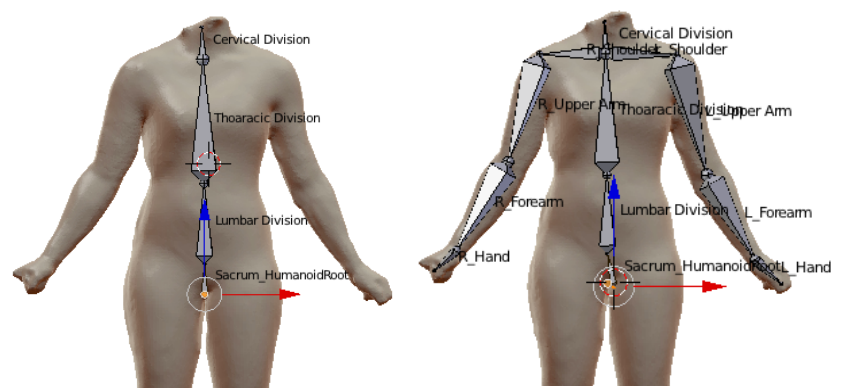

(a)

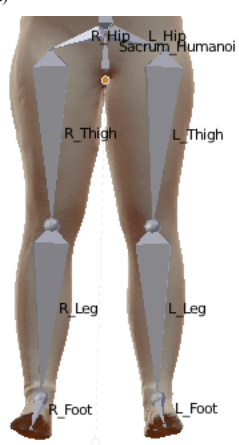

(c)

Figure 12: Skeleton construction regarding Blender's »Armature Modifier.
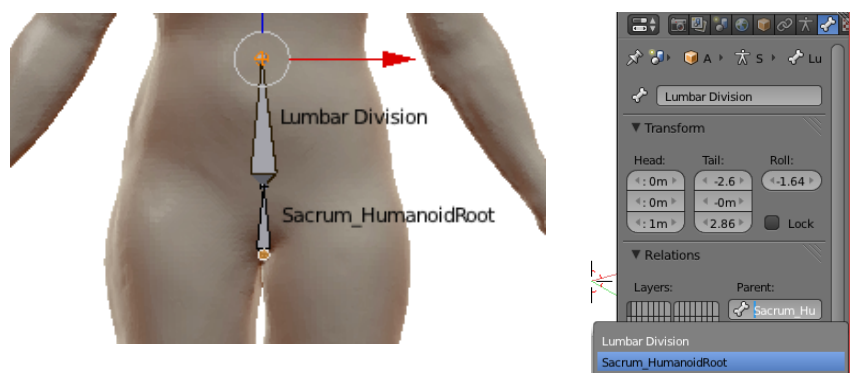

Figure 13: Establishing a »Parent-Child« relationship in »Edit Mode«. 
Citation: Kozar T, Rudolf A, Cupar A, Jevšnik S, Stjepanović Z (2014) Designing an Adaptive 3D Body Model Suitable for People with Limited Body Abilities. J Textile Sci Eng 4: 165. doi:10.4172/2165-8064.1000165

Page 6 of 13

\begin{tabular}{|c|c|}
\hline Anatomical feature point & Definition \\
\hline Crotch & The lowest part of the trunk, located on the head of the Sacrum_humanodid Root, between the Left- and Right legs. \\
\hline $\begin{array}{l}\text { Navel } \\
\text { (Belly Button Point) }\end{array}$ & $\begin{array}{l}\text { Located at the abdomen area, between the Abdominal extension and the Tenth Rib. It is placed on the bone tail of the Lumbar Division } \\
\text { bone }\end{array}$ \\
\hline $\begin{array}{l}\text { Cervical Vertebrae } \\
\text { (Vertebra Prominous) }\end{array}$ & $\begin{array}{l}\text { At the base of the neck portion of the spine and located at the tip of the spinous process of the } 7 \text { th cervical vertebra determined by } \\
\text { palpitation, often found by bending the neck or head forward. }\end{array}$ \\
\hline $\begin{array}{c}\text { Acromion } \\
\text { (Shoulder Point) }\end{array}$ & $\begin{array}{l}\text { The most prominent point on the upper edge of the acromial process of the shoulder blade (scapula) as determined by palpitation } \\
\text { (feeling). It is placed on the bone tails of Left- and Right shoulders. }\end{array}$ \\
\hline lliocristal & $\begin{array}{l}\text { Highest palpable point of the iliac crest of the pelvis, half the distance between the front (anterior) and back (posterior) upper (superior) } \\
\text { iliac spine. It is placed on the tails of the left- and right hip bones. }\end{array}$ \\
\hline Olecranon (Elbow) & $\begin{array}{l}\text { When an arm is bent, the furthermost (lateral) point of the olecranon which is the projection of the end of the innermost bone in the } \\
\text { lower arm (ulna); the joint between the upper and lower arm. It is placed on the tails of the Left and Right upper arm bones }\end{array}$ \\
\hline Carpus (Wrist) & $\begin{array}{l}\text { Joint between the lower arm and hand; Distal ends (toward the fingers) of the ulna (the innermost bone) and radius (the outermost } \\
\text { bone) of the lower arm. It is placed on the tails of the Left and Right Forearms. }\end{array}$ \\
\hline
\end{tabular}

Table 2: Anatomical features' locations and definitions [1, 29]

\begin{tabular}{|c|c|c|c|c|c|c|c|}
\hline \multirow{2}{*}{$\begin{array}{l}\text { Bone part } \\
\text { Bone name }\end{array}$} & \multicolumn{3}{|c|}{ Bone head location } & \multicolumn{3}{|c|}{ Bone tail location } & \multirow[b]{2}{*}{ Parent bone } \\
\hline & $\mathbf{x}$ & $\mathbf{y}$ & $\mathbf{z}$ & $\mathbf{x}$ & y & $\mathbf{z}$ & \\
\hline Sacrum_Humanoid Root & 0 & 23.2 & 18 & 0 & 23.2 & 1.18 & HumanoidRoot \\
\hline Lumbar Division & 0 & 23.2 & 1.18 & -2.5 & 38.7 & 2.93 & Sacrum_Humanoid Root \\
\hline Thoaracic Division & 2.58 & 38.7 & 2.93 & -2.5 & 62.1 & 6.41 & Lumbar Division \\
\hline Cervical Division & 2.58 & 62.1 & 6.41 & 0.42 & -32.3 & 7.28 & Thoaracic Division \\
\hline$L^{*} \_$Hip & 0 & 23.2 & 1.18 & 1.39 & 23.2 & 71.5 & Sacrum_Humanoid Root \\
\hline L_Thigh & 1.39 & 23.2 & 71.5 & 1.11 & 1.55 & -3.5 & L_Hip \\
\hline L_Leg & 1.11 & 1.55 & 3.5 & 1.21 & 1.55 & -7.6 & L_Thigh \\
\hline L_Foot & 1.21 & 1.55 & 7.60 & 1.3 & -6.67 & -8.2 & L_Foot \\
\hline $\mathrm{R}^{* *}$ Hip & 0 & 23.2 & 1.18 & -1.2 & 23.2 & 74.1 & Sacrum_HumanoidRoot \\
\hline R_Thigh & 1.29 & 23.2 & 74.1 & -1.2 & 23.2 & -3.4 & R_Hip \\
\hline R_Leg & 1.26 & 23.2 & 3.47 & -1.5 & 23.2 & -7.6 & R_Thigh \\
\hline R_Foot & 1.52 & 23.2 & 7.6 & -1.7 & 54.2 & -8.30 & R_Leg \\
\hline L_Shoulder & 2.58 & 62.1 & 6.41 & 2.05 & 29.7 & 6.19 & Thoaracic Division \\
\hline L_Upper Arm & 2.05 & 29.7 & 6.19 & 2.74 & 29.7 & 2.81 & L_Shoulder \\
\hline L_Forearm & 2.74 & 29.7 & 2.81 & 4.18 & 3.88 & 54.1 & L_Upper Arm \\
\hline L_Hand & 4.18 & 3.88 & 54.1 & 4.88 & 3.99 & -13.0 & L_Forearm \\
\hline R_Shoulder & 2.58 & 62.1 & 6.41 & -1.9 & 19,0 & 6.23 & Thoaracic Division \\
\hline R_Upper Arm & 1.96 & 19.0 & 6.23 & -2.9 & 14.7 & 2.91 & R_Shoulder \\
\hline R_Rorearm & 2.94 & 14.7 & 2.91 & -4.5 & -41.2 & 56.0 & R_Upperarm \\
\hline R_Hand & 4.54 & 41.2 & 56.0 & -4.8 & -36.9 & 28.8 & R_Forearm \\
\hline
\end{tabular}

Table 3: The bone locations in a standing position

$L^{*}$ - left

$\mathrm{R}^{* *}-$ Right

The bone locations in a standing position are summarized in Table 2 with a given "Parent bone" relationship.

The Blender also supports an "Inverse Kinematics" tool for enabling advanced animation and rendering. The "Bone Constrain" modifier was used to set the position of the last bone within the bone-chain, where other bones are positioned automatically. In order to connect the mesh to the skeleton, we applied the tool "Rotation and Scale" and then created a "Parent-Child relationship" between the mesh and the skeleton using the tool "With automatic weights". After the mentioned procedure, the prepared 3D body model was ready to perform posture animation for different poses.

\section{D body measurement analyses}

The objective of the research work was to test the accuracies and reliabilities of the 3D body models in both standing and sitting positions, as obtained by the scanning system GOM Atos II 400. In addition, we also tested the accuracy of a generalized adaptive $3 \mathrm{D}$ body

model, developed from the reconstructed 3D body model in a standing position. An investigation of anthropometric measurements was carried out in order to test the 3D body model's anthropometry in comparison with the real human's body measurements. Great importance was placed upon the adaptive 3D body model and its anthropometric behavior during posture adaptation. Therefore, we investigated 11 body measurements, which are defined in the standard ISO 8559 for garment construction and anthropometric surveys [32]. The definitions and locations of the body measurements are described in Table 3.

Traditional body measuring and virtual extraction of body measurements were adequately performed by taking important body landmarks into consideration. After measuring the human body in both standing and sitting postures, we performed three-dimensional body measurements' analysis, using the Rhinoceros 3D software program. Rhinoceros is 3D NURBS (Non-Uniform Rational B-Splines) curve, surface and solid modeler for Windows. It represents the primary modeling tool for designers of free-form physical shapes whose primary 

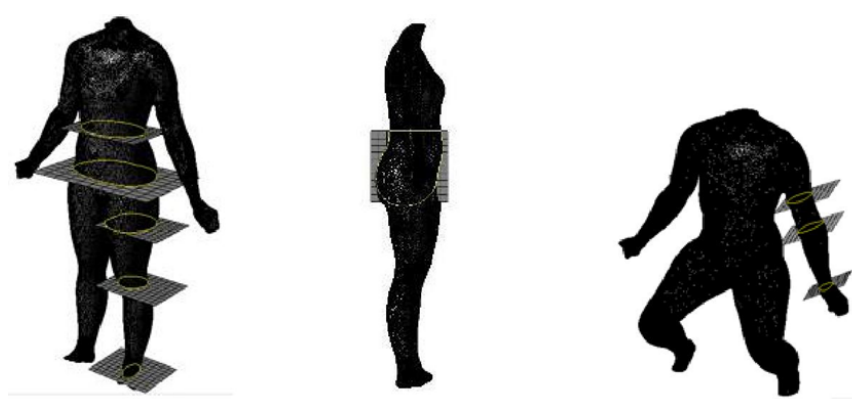

Figure 14: Virtual extraction of body measurements in different poses, using the software Rhinoceros 3D.

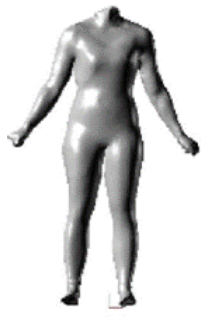

(a)
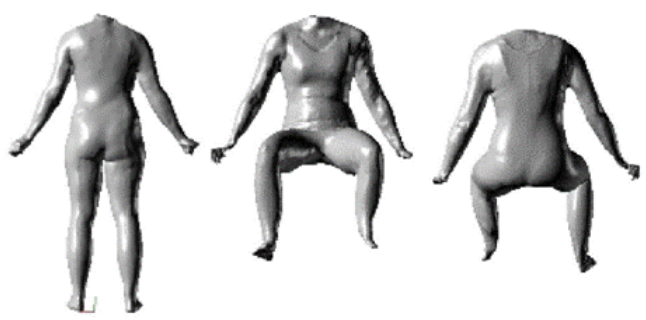

(b)
Figure 15: Reconstructed 3D body models from the front and back views standing (a) and sitting position (b).
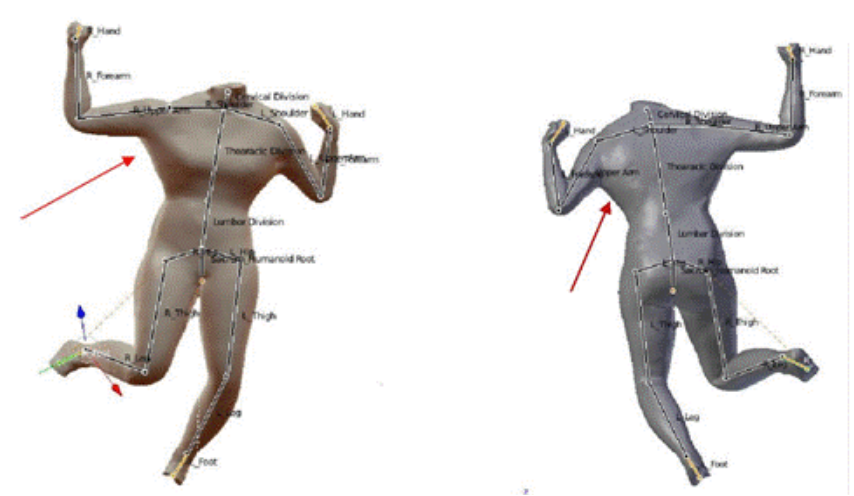

Figure 16: Mesh difficulties after posture adaptation.

goal is to design a human experience. It offers virtual measuring tools that provide accurate information about the objects [33]. In our study we used different analysis commands like the "Length" command for measuring different circumferences and lengths/distances of a $3 \mathrm{D}$ body model. By creating several rectangular planar surfaces at landmark locations of the body, we intersected meshes in order to extract the 3D body circumferences. Figure 14 shows the 3D body models, imported in the form of .stl files into the modeler for extracting the body measurements.

\section{Results and Discussion}

For achieving applicable 3D body models in both standing and sitting positions, we performed several activities within the course of this research: digitizing the surface of a tested person's body, using the optical scanning system GOM Atos II 3D, accurate surface reconstruction procedures for obtaining an optimal surface description of a scanned body and developing a generalized adaptive 3D body model for the purpose of posture adaptation.

\section{D body scanning and reconstructions of body models}

The scanning procedure was performed to digitize the entire body for each particular position. Difficulties did occur at overlapped regions which were difficult to capture, for example at regions belong to the armpits, knees and crotch. Therefore, careful surface processing was required for improving the surface descriptions of the meshes, which refers to section 4.2. Figure 15 shows the resulted 3D body models, scanned in both standing and sitting positions, imported into the program Rhinoceros 3D to determine 3D body measurements.

\section{Design of a kinematic skeleton}

For the purpose of this study we developed a kinematic skeleton consisting of 20 bones and 15 joints. The skeleton can be used for creating the digital 3D body models representing those people with limited body abilities. It was firstly evident that the mesh did not deform adequately and proportionally. Although each vertex group of the mesh were attached to the skeleton bones previously, difficulties regarding mesh stretching appeared, for example, at the vertex groups between the upper arms and the torso area, Figure 16. Blender's "Weight modifier" was used to increase the bone influence on the corresponding mesh-vertices and finally their influence on body measurements during posture adaptation

We altered the effect of the bones on vertices using a method called "Weight Painting". This method sustains a large amount of weight information in a very intuitive way. It was also used for rigging the meshes, where the vertex groups define the relative bone influence on the mesh. The selected mesh is shown slightly shaded with a rainbow color spectrum, where each color visualizes the weights associated to each vertex within the active vertex group. The weight is literally painted on the mesh surface by using a set of "Weight brushes", where the weights are visualized by a cold-hot color system [34]. By marking a bone in the "Weight modifier", the influence of a bone on the corresponding vertices can be concluded through the color-gradients. Areas with low influence are displayed in blue colors at cold areas, with weights close to the rate 0.0 . Areas with high influence are displayed in red colors as hot areas, with weights up to the rate 1.0. For example, the bone "Sacrum_HumanoidRoot" in default at the crotch area exhibits low influence on the corresponding vertices with a blue-green colorspectrum and weights' ratings from 0.1 to 0.3 , Figures $17 \mathrm{a}$ and $17 \mathrm{~b}$ show an average influence of the left forearm on the vertices at the elbow area. A green color-spectrum depicts weights ranging up to 0.5 , while a red color-spectrum shows high influence on the vertices of the left forearm. Vertices of the mesh at the torso area depict also a blue-green colorspectrum, when marking the "Thoracic Division" bone to display its influence on the correspondent vertices (Figure 17c).

In order to improve an adequate mesh deformation we chose different weights of the vertices to affect them regarding a particular bone. Their influence ranged from 0.5 to over 0.8 to avoid the robustness of mesh-deforming and the resulting difficulties of meshstretching and its sharp bending. Figure 18a shows the difficulties of mesh stretching at the breast circumference. Therefore, we altered the effect of the "Thoracic Division" bone on the related vertex group by using a weight influence of 0.8 and acquired a slight mesh deformation in Figure 18b. The same procedure remained ineffective for the mesh at the right side shown in Figure 18c. Namely, the mesh in this area 


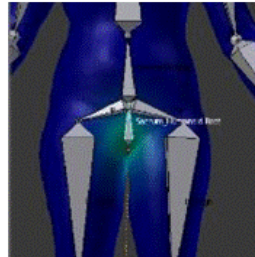

(a)

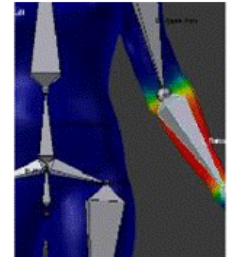

(b)

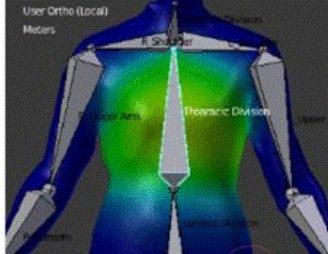

Figure 17: Color-spectra of different vertex groups for displaying the bone influence. Color-spectra of different vertex groups for displaying the bone influence.

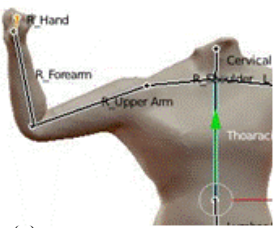

(a)

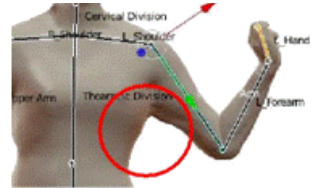

(c)

Figure 18: Using Blender's "Weight modifier" tool to improve adequate mesh deformation at The armpits.
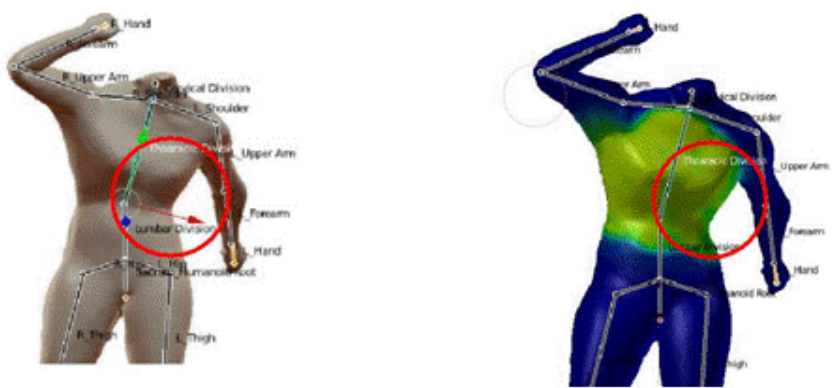

Figure 19: Using Blender's "Weight modifier" tool to improve adequate mesh deformation at the waist circumference.

was not credible for the real surface of the body, caused by the complex unification of individual scans into a global co-ordinate system and further incomplete surface reconstruction in this area.

\section{Towards a generalized adaptive 3D body model}

During the course of this research we developed a generalized adaptive 3D body model based on the previously designed kinematic skeleton. The purpose of an adaptive 3D body model is to enable a reliable and fast adaptation to the body measurements and posture specificities of real persons - people with limited body abilities. For this reason, some further adaptations of the developed body model were needed.

In order to avoid sharp bending of the mesh at the waist circumference, we increased the influence of the "Thoracic Division" and "Lumbar Division" bones to 0.5 weight in order to create a gently mesh-deformation after adapting the 3D body model to different positions (Figure 19).

Figure 20 displays the altered effect of the "Left Thigh" bone on the related vertex group at this area. We used weight influences ranging from 0.6 to 0.8 to create a smooth mesh-deformation and consequently to improve the thigh circumference measurement

\section{Analysis of the differences of body measurements caused by posture change}

One of the goals of this study was to test the 3D body model's accuracy regarding both standing and sitting positions, and the suitability of the developed 3D body model the posture adaptation of which had been improved by upgrading the kinematic skeleton structure inside the watertight mesh of a 3D body model in a standing position. The adapted 3D body model in a sitting position, which is equal to the sitting posture of a tested person during the scanning procedure, is shown in Figure 21.

We compared the body measurements of a real person with the corresponding 3D digitized body model and determined their differences in square centimeters and percentages. In general, the 3D

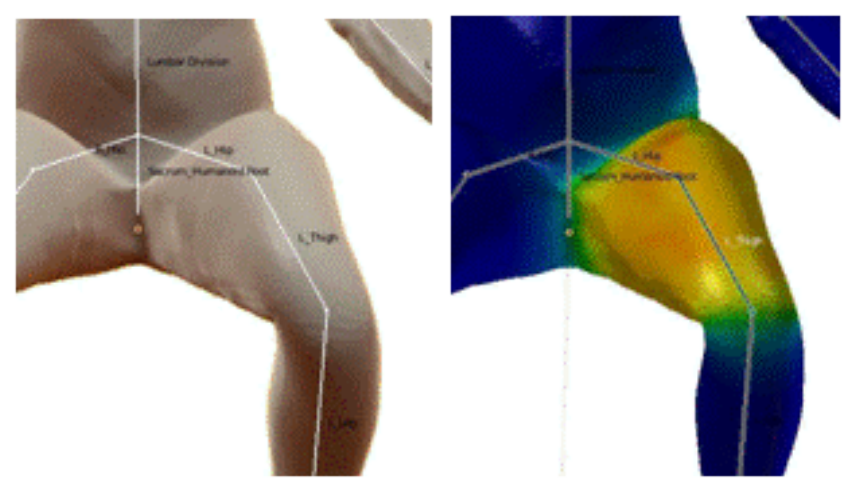

Figure 20: Using Blender's "Weight modifier" tool to improve adequate mesh deformation at the thigh circumferences.

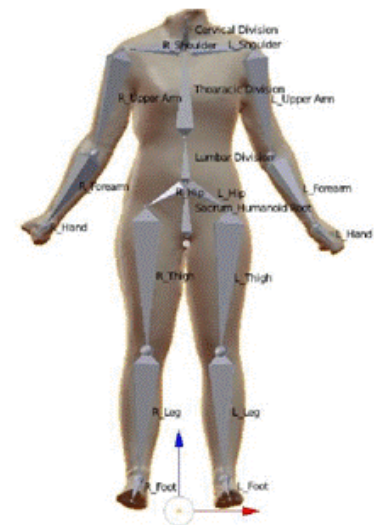

(a)

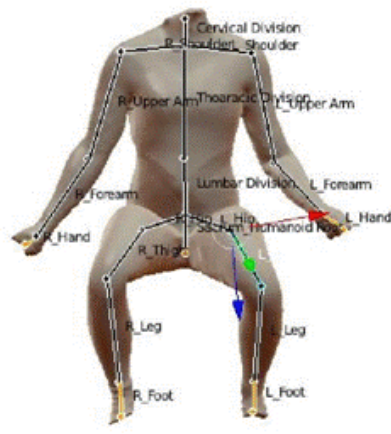

(b)
Figure 21: 3D body model posture adaptation in a standing position with an octahedral skeleton presentation (a) and adaptation to a sitting position with a stick representation of a skeleton (b). 
Citation: Kozar T, Rudolf A, Cupar A, Jevšnik S, Stjepanović Z (2014) Designing an Adaptive 3D Body Model Suitable for People with Limited Body Abilities. J Textile Sci Eng 4: 165. doi:10.4172/2165-8064.1000165

Page 9 of 13

\begin{tabular}{|c|c|}
\hline Horizontal measurements & Body measurements' definitions \\
\hline Breast circumference & $\begin{array}{l}\text { The maximum horizontal circumference measured during normal breathing with the subject standing upright and the tape-measure } \\
\text { passed over the shoulder blades (scapulae), under the armpits (axillae), and across the breast nipples. }\end{array}$ \\
\hline $\begin{array}{l}\text { Under breast } \\
\text { circumference }\end{array}$ & The horizontal girth of the body just below the breasts. \\
\hline $\begin{array}{l}\text { Waist } \\
\text { circumference }\end{array}$ & $\begin{array}{l}\text { The circumference of the natural waistline between the top of the hip bones (iliac crests) and the lower ribs, measured with the } \\
\text { subject breathing normally and standing upright }\end{array}$ \\
\hline $\begin{array}{l}\text { Hips } \\
\text { circumference }\end{array}$ & $\begin{array}{l}\text { The horizontal circumference measured round the buttocks at the level of the greatest lateral trochanteric projections, with the } \\
\text { subject standing upright. }\end{array}$ \\
\hline $\begin{array}{l}\text { Thigh } \\
\text { circumference }\end{array}$ & The horizontal circumference measured without constriction, at the highest thigh position, with the subject standing upright. \\
\hline Knee circumference & $\begin{array}{l}\text { The circumference of the knee measured with the subject standing upright and the upper border of the tape-measure at the tibia } \\
\text { level. }\end{array}$ \\
\hline $\begin{array}{l}\text { Calf } \\
\text { circumference }\end{array}$ & $\begin{array}{c}\text { The maximum circumference of the calf measured with the subject standing upright, legs slightly apart, and with the body mass } \\
\text { equally distributed on both legs. }\end{array}$ \\
\hline Upper arm circumference & $\begin{array}{l}\text { The maximum circumference of the upper arm at lowest scye level, measured with the subject standing upright with arms hanging } \\
\text { naturally. }\end{array}$ \\
\hline Vertical measurements & Body measurements' definitions \\
\hline Front waist length & The distance from the neck shoulder point, over the nipple, then vertically straight to the front waist. \\
\hline Cervical to breast point & The distance from the 7th cervical vertebra, round the base of the neck, to the nipple. \\
\hline Total crotch length & $\begin{array}{c}\text { The distance, measured using the tape-measure, from the center of the natural waist level at the front of the body, over the crotch, } \\
\text { to the center of the waist level. }\end{array}$ \\
\hline Back waist length (Cervical to waist) & $\begin{array}{l}\text { The distance, measured using the tape-measure, from the 7th cervical vertebra, following the contour of the spinal column, to the } \\
\text { waist. }\end{array}$ \\
\hline Arm length /shoulder to wrist) & The distance, measured using the tape-measure, from the armscye/shoulder line intersection (acromion) to the elbow \\
\hline Outside leg length & The distance from the waist to the ground measured using the tape-measure following the contour of the hip, then vertically down. \\
\hline
\end{tabular}

Table 4: Body measurements' definitions and measuring locations [32]

\begin{tabular}{|c|c|c|c|c|}
\hline & Test & 3D body model/ & Difference & Percentage \\
\hline & person/ & standing & between body & Difference \\
\hline \multirow[t]{2}{*}{ Body measures } & standing & position & measurements & $(\%)$ \\
\hline & position & & $(\mathrm{cm})$ & \\
\hline Breasts circ. & 88.50 & 88.78 & 0.28 & 0.24 \\
\hline Under breasts circ. & 79.80 & 80.28 & 0.48 & 0.38 \\
\hline Waist circ. & 73.20 & 73.51 & 0.31 & 0.26 \\
\hline Hips circ. above thigh & 102.20 & 103.38 & 1.18 & 1.21 \\
\hline Thigh circ. & 61.30 & 61.62 & 0.32 & 0.19 \\
\hline Knee circ. & 41.30 & 41.65 & 0.35 & 0.14 \\
\hline Calf circ. & 39.29 & 38.78 & -0.51 & -0.20 \\
\hline Upper arm circ. & 27.76 & 29.20 & 1.44 & 0.4 \\
\hline Front waist Length & 46.50 & 46.95 & 0.45 & 0.21 \\
\hline Cervical to Breast point & 26.50 & 26.68 & 0.18 & 0.05 \\
\hline Total crotch length & 72.80 & 73.22 & 0.42 & 0.31 \\
\hline Back waist length & 41.76 & 41.95 & 0.19 & 0.08 \\
\hline Arm length & 57.00 & 57.60 & 0.60 & 0.35 \\
\hline Outside leg length & 103.80 & 104.10 & 0.30 & 0.31 \\
\hline
\end{tabular}

Table 5: Differences in body measurements at a standing position.

body model exhibited primarily increased circumferences and bodylengths. The measured differences between standing posture bodies (Table 4 ) ranged from $-0.2 \%$ to $1.21 \%$. Hips' circumferences showed the greatest difference, namely $1.18 \mathrm{~cm}(1.21 \%)$, while calf circumferences showed the lowest differences, decreasing for $0.51 \mathrm{~cm}(-0.2 \%)$ due to difficult processes of alignment for individually observed meshes into one independent mesh when observing $3 \mathrm{D}$ body meshes using the GOM Atos optical scanning system. These were followed by breasts, under the breasts, and waist circumferences which showed differences of not even $0.5 \mathrm{~cm}$. For body lengths we obtained the best result for the length at cervical to breast point, with a difference for not even $0.2 \mathrm{~cm}$, followed by back waist length with a difference of $0.19 \mathrm{~cm}$ and outside leg length with a difference of $0.3 \mathrm{~cm}$.

Three-dimensional body measurement differences of a 3D body model in a sitting position shown in Table 5 is ranged from $-0.43 \%$ to $2.03 \%$. Even in this case, hips-circumference showed the greatest difference of $1.89 \mathrm{~cm}(2.03 \%)$, while the calf circumference decreased by $1.1 \mathrm{~cm}(-0.43 \%)$, as a result of aligning individual scans into one global coordinate system. We obtained slightly increased results for breast and waist circumference than in the previous case but without $0.5 \mathrm{~cm}$ to achieve. Total crotch length differed by $1.17 \mathrm{~cm}(0.85 \%)$ due to difficult scanning of overlapped regions and further surface reconstruction. Back waist length showed the best result, namely a difference of $0.21 \mathrm{~cm}$, followed by outside leg length with a difference of $0.21 \mathrm{~cm}$ when comparing the two types of measurements.

The differences in the 3D skeletal body model's measurements (Table 6), ranged from $-0.73 \%$ to $0.78 \%$. It was evident that the waist circumference had decreased by $0.99 \mathrm{~cm}(0.73 \%)$ while the hip 
Citation: Kozar T, Rudolf A, Cupar A, Jevšnik S, Stjepanović Z (2014) Designing an Adaptive 3D Body Model Suitable for People with Limited Body Abilities. J Textile Sci Eng 4: 165. doi:10.4172/2165-8064.1000165

Page 10 of 13

\begin{tabular}{|c|c|c|c|c|c|c|c|}
\hline \multirow[t]{2}{*}{ Body measurements } & \multirow[t]{2}{*}{$\begin{array}{l}\text { Test person/sitting } \\
\text { position }\end{array}$} & \multirow{2}{*}{ 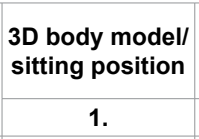 } & \multirow{2}{*}{$\begin{array}{c}\text { 3D adaptive } \\
\text { body model } \\
2 .\end{array}$} & \multicolumn{2}{|c|}{$\begin{array}{c}\text { Differences } \\
\text { between body measurements } \\
(\mathrm{cm})\end{array}$} & \multicolumn{2}{|c|}{$\begin{array}{l}\text { Percentage difference } \\
(\%)\end{array}$} \\
\hline & & & & 1. & 2. & 1. & 2. \\
\hline Breasts circ. & 88.2 & 88.57 & 88.72 & 0.37 & 0.52 & 0.33 & 0.46 \\
\hline Under breasts circ. & 80.56 & 80.71 & 80.3 & 0.15 & -0.24 & 0.12 & -0.19 \\
\hline Waist circ. & 74.5 & 75.10 & 73.51 & 0.60 & -0.99 & 0.45 & -0.73 \\
\hline Hips circ. above thigh & 107.49 & 109.38 & 108.22 & 1.89 & 0.73 & 2.03 & 0.78 \\
\hline Thigh circ. & 60.20 & 60.40 & 60.54 & 0.20 & 0.34 & 0.12 & 0.2 \\
\hline Knee circ. & 40.40 & 42.20 & 41.84 & 1.8 & 1.44 & 0.72 & 0.58 \\
\hline Calf circ. & 39.29 & 38.2 & 38.78 & -1.1 & -0.51 & -0.43 & -0.2 \\
\hline Upper arm circ. & 27.76 & 28.75 & 28.63 & 0.9 & 0.87 & 0.27 & 0.24 \\
\hline Front waist Length & 45.2 & 45.57 & 46.50 & 0.37 & 1.3 & 0.17 & 0.58 \\
\hline Cervical to Breast point & 26.34 & 26.67 & 26.6 & 0.33 & 0.26 & 0.08 & 0.06 \\
\hline Total crotch length & 73.33 & 74.50 & 73.22 & 1.17 & -0.11 & 0.85 & -0.08 \\
\hline Back waist length & 41.26 & 41.47 & 41.64 & 0.21 & 0.38 & 0.08 & 0.16 \\
\hline Arm length & 57.00 & 57.43 & 57.60 & 0.43 & 0.6 & 0.25 & 0.35 \\
\hline Outside leg length & 104.48 & 104.70 & 104.28 & 0.22 & -0.2 & 0.23 & -0.21 \\
\hline
\end{tabular}

Table 6: Differences in body measurements in a sitting position.

\begin{tabular}{|c|c|c|c|c|c|c|c|c|c|c|}
\hline Body measurements & TP & SD & $3 \mathrm{D} \mathrm{BM}_{\mathrm{A}}$ & SD & $F_{c}$ & $F_{t}$ & $H_{0}: \sigma_{1}^{2}=\sigma_{2}^{2}$ & $t_{c}$ & $t_{t}$ & S.D. \\
\hline Breasts circ. & 88.2 & 2.39 & 88.72 & 1.51 & 1.58 & 6.451 & Confirmed & 0.064 & 2.878 & No \\
\hline Under breasts circ. & 80.56 & 0.04 & 80.3 & 0.06 & 1.5 & 6.451 & Confirmed & 2.26 & 2.878 & No \\
\hline Waist circ. & 74.5 & 0.438 & 73.51 & 0.0297 & 14.7 & 6.451 & Rejected & 0.05 & 2.878 & No \\
\hline Hips circ. above thigh & 107.49 & 0.287 & 108.22 & 0.35 & 1.21 & 6.451 & Confirmed & 7.00 & 2.878 & Yes \\
\hline Thigh circ. & 60.20 & 0.074 & 60.54 & 0.18 & 2.4 & 6.451 & Confirmed & 2.23 & 2.878 & No \\
\hline Knee circ. & 40.40 & 0.11 & 41.84 & 0.174 & 1.58 & 6.451 & Confirmed & 0.96 & 2.878 & No \\
\hline Calf circ. & 39.29 & 0.019 & 38.78 & 0.206 & 10.8 & 6.451 & Rejected & 0.2 & 2.878 & No \\
\hline Upper arm circ. & 27.76 & 0.085 & 28.63 & 0.015 & 5.6 & 6.451 & Confirmed & 1.77 & 2.878 & No \\
\hline Front waist Length & 45.2 & 0.033 & 46.50 & 0.067 & 2.0 & 6.451 & Confirmed & 1.87 & 2.878 & No \\
\hline Cervical to Breast point & 26.34 & 0.057 & 26.6 & 0.085 & 1.49 & 6.451 & Confirmed & 0.85 & 2.878 & No \\
\hline Total crotch length & 73.33 & 0.052 & 73.22 & 0.015 & 3.5 & 6.451 & Confirmed & 1.18 & 2.878 & No \\
\hline Back waist length & 41.26 & 0.033 & 41.64 & 0.012 & 2.75 & 6.451 & Confirmed & 2.08 & 2.878 & No \\
\hline Arm length & 57.00 & 0.028 & 57.60 & 0.0179 & 4.35 & 6.451 & Confirmed & 2.02 & 2.878 & No \\
\hline Outside leg length & 104.48 & 0.31 & 104.28 & 0.038 & 8.15 & 6.451 & Rejected & 1.67 & 2.878 & No \\
\hline
\end{tabular}

Table 7: Descriptive statistics to determine significant differences between the observed measurements.

TP: test person

3D $\mathrm{BM}_{\mathrm{A}}: 3 \mathrm{D}$ adaptive body model

SD: Standard deviation

Fc: F-value calculated

Ft: F- tabular value

$\sigma_{1}^{2}, \sigma_{2}^{2}$ : sums of square deviations of the two groups of measurements

$H_{0}: \sigma_{1}^{2}=\sigma_{2}^{2}:$ Hypothesis

$t_{c}:$ t-values calculated

$t_{t}:$ t-tabular value

S.D.: Significant difference

circumference had increased by about $0.73 \mathrm{~cm}(0.78 \%)$, and thus confirmed a better result than in the previous case. The obtained measurements of the breasts' circumferences exhibited a slightly higher result of $0.52 \mathrm{~cm}(0.46 \%)$, while the results for under the breast, knee and upper arm circumferences confirmed an improvement in the 3D skeletal model's measurements in comparison with traditional ones. The body length at the cervical to the breast point increased by just $0.26 \mathrm{~cm}(0.06 \%)$ and confirmed a better result than in the previous case. A small decreased difference appeared for the total crotch length, namely $0.11 \mathrm{~cm}(0.08 \%)$, which confirmed a better result as well. Outside leg length decreased for $0.2 \mathrm{~cm}$ which is comparable to previous result of a 3D body model in a sitting position. We obtained also slightly increased differences for back waist length, namely a difference of $0.38 \mathrm{~cm}$, followed by arm length with a difference of $0.6 \mathrm{~cm}$, which is slightly higher as in previous case.
In order to test the suitability of the generalized adaptive $3 \mathrm{D}$ body model developed from the reconstructed 3D body model in a standing position, descriptive statistics of real human's measurements in comparison with 3D body model's measurements were analyzed to determine significant differences between the observed measurements by $\mathrm{F}$ - and $\mathrm{t}$ - test (Table 7).

Analysis of the difference between real human's measurements in comparison with 3D body model's measurements using tests, such as F-test and t-test, revealed a significant difference in hips circumference above thigh only.

It can be concluded that the improved body measurements of a generalized adaptive 3D body model resulted from the upgrading its kinematic skeleton and the use of virtual mesh-deformation modeling tools like Blender's "Mesh modifier" tool. It is possible to adapt the 

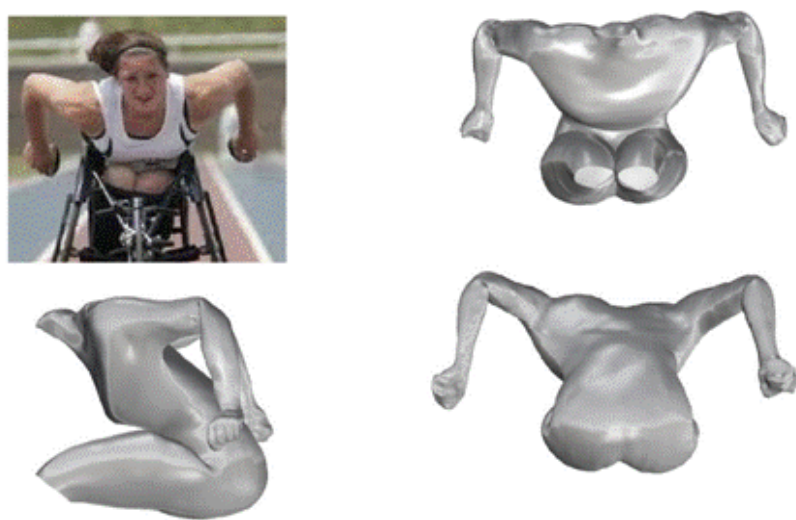

Figure 22: 3D body model adaptation to a wheelchair racing athlete.
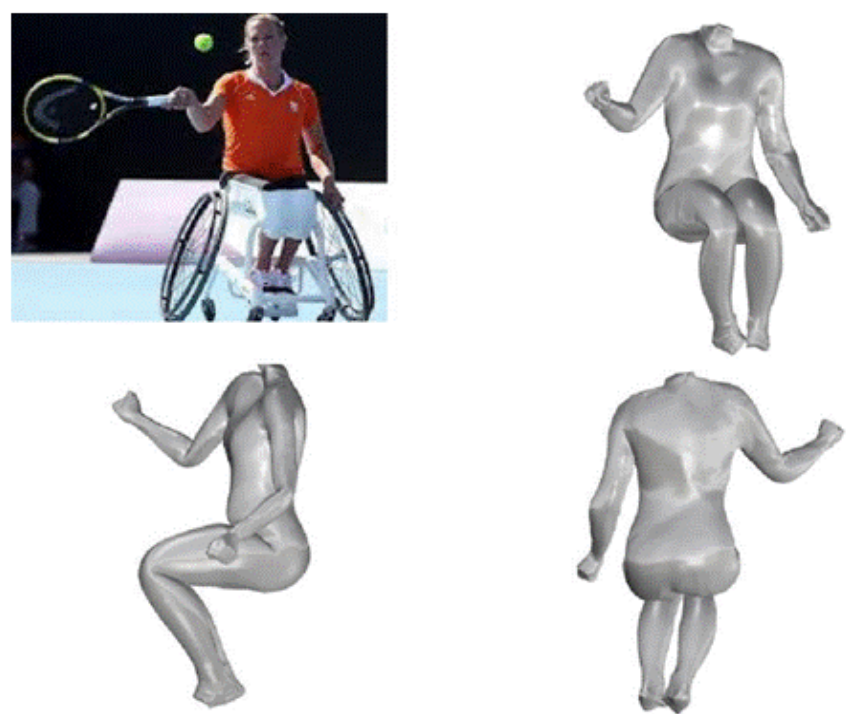

Figure 23: 3D body model adaptation to a wheelchair tennis player.

observed 3D body model to different positions while sustaining body measurements as in real-life.

It is well-known that people with limited body abilities achieve greater results in sports and in other social activities. In sport especially they compete professionally at the highest levels at international competitions supported by organizations like the International Wheelchair and Amputee Sports Federation (IWAS) in the United Kingdom [35] or the Association for disability sports in Slovenia [36]. In order to provide comfortable and aesthetic sportswear for this group of people of the world's population, there is a demand for supplying garments virtually prototyped using a general $3 \mathrm{D}$ body model for nonstandard body shape characteristics, adaptable for different bodies' positions?

Further, the efficiency of mesh-deforming was determined after adapting a 3D body model to images of people with limited body abilities occupying positions while playing sports games. Figure 22 shows an adapted 3D body model to a female athlete with amputated legs during wheelchair racing, which represents one of the more exciting Paralympics disciplines [37]. The 3D body model was completely adapted to the desired position. Incorrect mesh-deforming occurred in the elbow area and could not be corrected with the "Mesh modifier" tool.

Figure 23 shows a female player during wheelchair tennis at the Paralympics Games of the year 2012 [38]. Again, the 3D body model was completely adapted to the desired position but again with incorrect mesh-deformation occurring in the elbow area. These problems can be solved by manual correction procedures with the majority of $3 \mathrm{D}$ modelers.

Figures $24 \mathrm{a}$ and $24 \mathrm{~b}$ show two additional examples of adapting the generalized 3D body model to different positions, for example to the paraplegic female basketball player [39], (Figure 24a) or even to the athlete with an amputated leg [40], (Figure 24b).

It is evident, that in spite of carefully carried out scanning and mesh processing procedures, difficulties from incorrect mesh-deformations remained during posture adaptation. Some of them could be corrected with the "Mesh modifier" tool through altering the effect of the bones on the correspondence mesh vertices. Other difficulties remain less consistent with real humans' movements, especially in the elbow area, where the bone of the kinematic skeleton was imprecisely attached to the feature points. Additional adjustments to kinematic skeleton constructions at exact feature points' areas are therefore necessary.

In order to show the usefulness of the observed 3D body models, we performed garments' pattern designing using two different systems for this purpose, namely Marvelous designer 3 and Optitex PDS. The observed 3D body model in a standing position was imported into a form of .dae file (Collada file) and then into the Marvelous designer program for performing tights and tunic pattern designing (Figure 25a). The Optitex Pattern Design System was used to construct T-Shirt and trouser patterns. The generalized adaptive 3D body model was imported in the form of a stl (Stereo Litography file) to simulate garments' appearances (Figure 25b).

It is evident that in spite of carefully carried out scanning and mesh processing procedures, difficulties of incorrect mesh-deformations remained during posture adaptations. Some of them could be corrected using the "Mesh modifier" tool through altering the effects of the bones on the correspondence mesh vertices. Other difficulties remain less consistent to real humans' movements, especially in the elbow area, where the bone of the kinematic skeleton was imprecisely attached to the feature points. Additional adjustments of a kinematic skeleton construction at exact feature points' areas are therefore necessary.

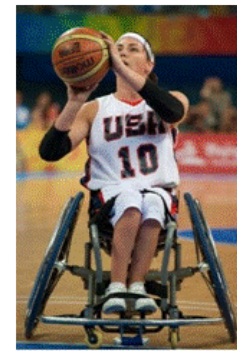

(a)

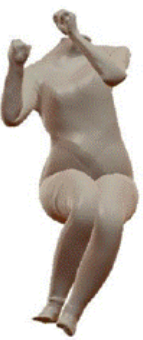

Figure 24: 3D body model adaptations to female athletes active in different sports.
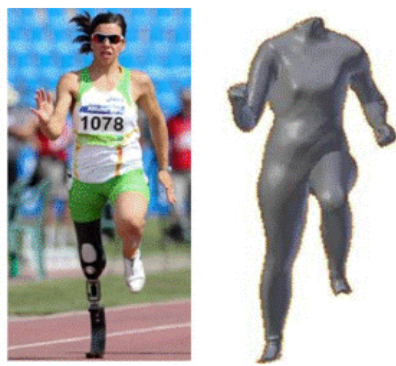


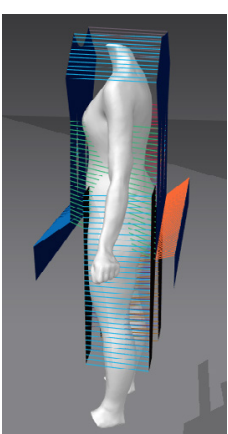

(a)

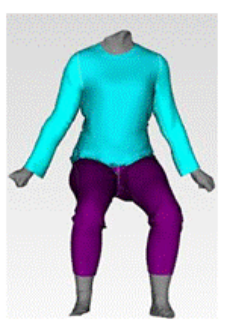

(b)

Figure 25: Garment pattern design using Marvelousdesigner 3 and Optitex PDS.

\section{Conclusions}

We have described an approach for developing a generalized adaptive 3D body model, suitable for people with limited body abilities, the positions of which could be adapted to different positions. An adequate mesh of a tested person's body in both standing and sitting positions was achieved using a general-purpose scanner GOM Atos II 400. Through pre-processing and post-processing procedures, the mesh was reconstructed using several graphic programs, such as GOM Inspect, MeshLab and Blender. Kinematic skeleton construction was carried out in order to provide posture adaptations to different positions. Further, incorrect mesh-deformations were improved through altering the effect of the bones on the corresponding meshvertices by using Blender's "Mesh modifier" tool. While the differences in body measurements between real persons and the adaptive 3D body model in a sitting posture confirmed the best results, consequently the "Mesh modifier" tool was ineffective when correcting mesh imperfections in the elbow areas, after adapting the 3D body model to images of people with limited body abilities. Due to the high densities of the observed polygonal meshed surfaces, their processing is difficult to approximate regarding real humans' behavior/movements. Future work will be focused on developing a meshed 3D body model, with lower densities of polygons in order to facilitate their processing for the purpose of posture adaptation. In the future, our research will focus on 3D body model parametrization to ensure 3D body model's fitting to body shapes, taken from the images. In this way, it will be possible to design appropriate parametric 3D body models, which will be digitized reflections of real persons with all individual characteristics required for virtual prototyping and virtual fitting of garments.

Within the course of this research we have developed a generalized adaptive $3 \mathrm{D}$ body model based on the previously designed kinematic skeleton. This developed generalized adaptive 3D body model will be used for a reliable and fast adaptation to the body measurements and posture specifics of real persons - people with limited body abilities.
Adapted body models, maximally personalized to mimic the real persons, will be imported into commercial CAD/PDS program packages and further tested for efficient garment prototyping and visualizations of two types of garments: special sports clothing and elegant dresses for people with limited body abilities. Both garment types are needed and desired by this special group of people. Our intention is to help them reach this goal.

\section{References}

1. Magnenat- Thalmann N, Thalmann D (2004) in "Handbook of Virtual Humans", John Wiley \& Sons Ltd., The Atrium, Southern Gate, Chichester, UK.

2. Mpampa ML, Azriadis NP, Sapidis NS (2010) A new methodology for the development of sizing systems for the mass customization of garments. International Journal of Clothing science and Technology 22:49-68.

3. Lectra (2012)Smart design, Design room,Lectra, France.

4. Marvelous Designer (2012) Marvelous designer, Korea.

5. Gerber Technology (2012) Product design, Apparel retail, Gerbertechnology, USA.

6. OptiTex (2012) Apparel, Optitex,USA.

7. Pilar T (2012) Development of 3D prototypes of women's clothing. Master's Thesis, University of Maribor, Faculty of Mechanical Engineering.

8. Lectra (2014) Lectra 3D technology is the centrepiece of the end-to-end development process, Press Release, France.

9. United Nations (2012) What is disability and who are persons with disabilities USA.

10. Gupta D (2011) Functional Clothing- Definition and Classification. Indian Journal of Fibre and Textile research 36: 321-326.

11. Seo H, Magnenat-Thalmann N (2004) An example- based approach to human body manipulation. Graphical Models 66: 1-23.

12. Zhengdong L, Shuyuan S (2010) Free-form deformation algorithm of human body model for garment. The 2010 International Conference on Computer Application and System Modelling 11: 602-605.

13. Allen B (2005) Learning body shape models from real-world data. Ph. D. Dissertation, University of Washington, USA.

14. Wang CCL (2005) Parameterization and parametric design of mannequins Computer-Aided Design 37: 83-98.

15. Hilton A, Beresford D, Gentils T, Smith R, Sun W (1999) Virtual People: Capturing human models to populate virtual worlds. Proc.Computer Animation 174-185.

16. Lee W, Gu J, Magnenat-Thalmann N (2000) Generating animatable 3D virtua humans from photographs. Proc. Eurographics ,2000, Computer Graphics Forum 19: 1-10

17. Bălan A (2010) Detailed human shape and Pose from images, Ph. D Dissertation, Brown University, USA.

18. Kozar T, Rudolf A, Jevšnik S, Stjepanović Z (2012) Developing the accurate sitting position 3D body model for garment prototyping. Proceedings of $4^{\text {th }}$ International Scientific Professional Conference Textile Science and Economy Zrenjanin 133-138.

19. Rudolf A, Kozar T, Jevšnik S, Cupar A, Drstvenšek I (2013) Research on 3D body model in a sitting position obtained with different 3D scanners. Istanbul Technical University, Faculty of Textile Technologies and Design 6.

20. Rudolf A, Kozar T, Cupar A, Jevšnik S, Stjepanović Z (2013) Development of appropriate garment pattern designs for a sitting position 3D body model. The $10^{\text {th }}$ Conference of Chemists, Technologists and Environmentalists of Republic of Srpska, University of Banja Luka, Faculty of technology 380.

21. Barbero BR, Ureta Santos E (2011) Comparative study of different digitization techniques and their accuracy. Computer-Aided Design 43: 188-206.

22. Brajlih T, Tasić T, Drstvenšek I, Valentan B, Hadžistević M, et al. (2011) Possibilities of Using Three-Dimensional Optical Scanning in Complex Geometrical Inspection. Strojniški vestnik - Journal of Mechanical Engineering 57: 826-833. 
Citation: Kozar T, Rudolf A, Cupar A, Jevšnik S, Stjepanović Z (2014) Designing an Adaptive 3D Body Model Suitable for People with Limited Body Abilities. J Textile Sci Eng 4: 165. doi:10.4172/2165-8064.1000165

Page 13 of 13

\section{GOM (2014) Optical Measuring Techniques, GOM, USA.}

24. Ma J (2011) Surface reconstruction from unorganized point cloud data via progressive local mesh matching. Ph. D .Dissertation, The University of Western Ontario, Canada.

25. GOM (2014) GOM Inspect software, GOM, USA.

26. MeshLab (2014) 3D - Coform project.

27. Kozar T, Rudolf A, Jevšnik S, Cupar A, Priniotakis G, et al. (2014) Accuracy evaluation of a sitting 3D body model for adaptive garment prototyping. Proceedings of AUTEX 2014 Conference, Bursa.

28. Petrak S (2007) The method of 3D garment construction and cutting pattern transformation models. Doctoral Dissertation, Faculty of Textile Technology, Zagreb.

29. H-Anim (2014) Humanoid animation ISO/IEC/FCD 19774:200x Specification.

30. Blender (2014) 3D animation suite, Blender, Netherlands.

31. Mazany O (2007) Articulated 3D human model and its animation for testing and learning algorithms of multi-camera systems. Maters's Thesis, Czech Technical University, Faculty of Electrical Engineering, Czech Republic.
32. International Standard (1989) Garment construction and anthropometric surveys- Body dimensions, International Standard ISO 8559 , Switzerland.

33. McNeel R (2002) Rhinoceros Version 3.0.: Nurbs modeling for Windows.

34. Weight Paint mode

35. Weight paint mode (2014) Meshses, Modelling manual.

36. IWAS (2014) International Wheelchair and Amputee Sports Federation, UK. 37. NSIOS (2014) Association for the disability sports, Slovenia.

38. Paralympic Disciplines (2014) Paralympic organization, Germany.

39. Paralympics (2012) Wheelchair tennis winner Esther Vergeer does it again Germany.

40. Paralympic (2014) Taxonomy, London.

41. $A B C$ radio national (2014) Bionic athlete, Body sphere, $A B C$ radionational, Australia. 